\title{
An Electronic Method for Measuring the Fit of Removable Partial Denture Frameworks to Dental Casts
}

\author{
Robert J Williams*, Tahseen Rafik and Zeid Al-Hourani \\ Centre for Dental Technology and School of Product and Engineering Design, University of Wales Institute, Cardiff, Llandaff, \\ Cardiff, CF5 2YB, United Kingdom
}

Received 10 January 2007; accepted 9 May 2007

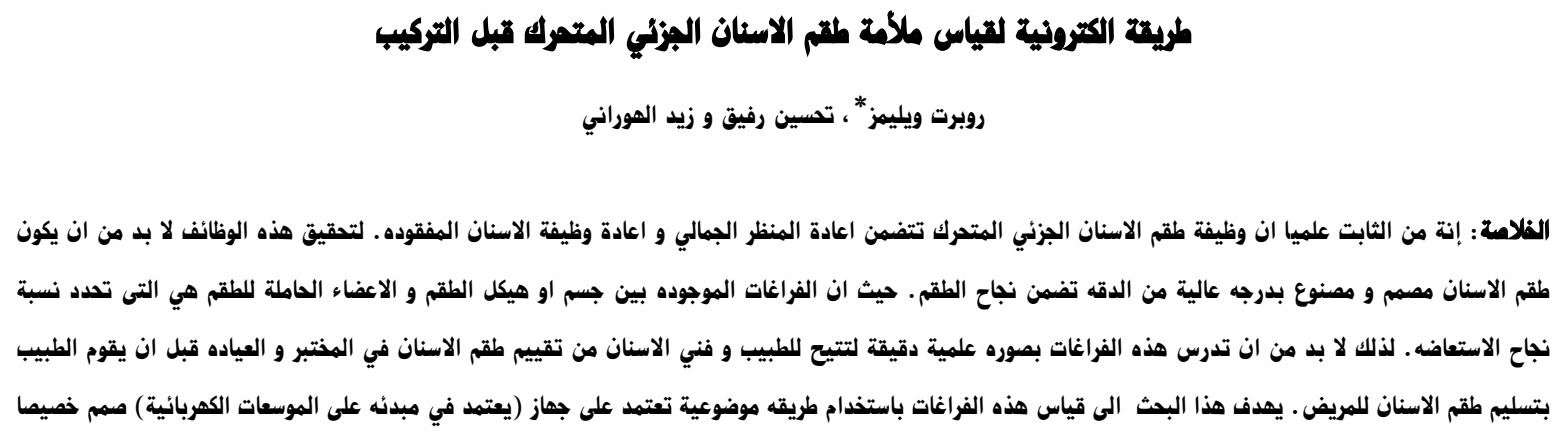

لهذه الغاية.

الهلمات الهنتامية: تقنيات الاسنان، اطقم الاسنان الجزئية المتحركة، الهبسات ، قياس الفجوات الصغيره.

\begin{abstract}
It is well established that the Removable Partial Denture (RPD) is an effective treatment prosthesis. The objectives of a successful RPD are: to preserve the health of remaining oral structure, restore function and restore esthetics. To achieve these objectives, an RPD framework must fit accurately to the supporting structures. This paper presents a method for measuring the gaps or spaces present between the RPD framework and supporting structures which will enable the dentist and the dental technician to evaluate the accuracy of fitting of the prosthesis before it is delivered to the patient. The method used in this research is based on the principle of electric capacitance and uses a specially designed prototype measurement system.
\end{abstract}

Keywords: Dental technology, Capacitance transducers, Small gap measurement

\section{Introduction}

It is widely acknowledged that in order for a removable partial denture (RPD) framework to achieve maximum function, create pleasing aesthetics and remain biocompatible, accuracy of fit must be optimized (Sykora, 1997; Brudvik, et al. 1992 and Stern, et al. 1985). RPD frameworks are fabricated from high-shrinkage metal alloy, leading to difficulties in achieving accurate fitting (Fritell, et al. 1985). In order to determine how to hone fabrication processes so that accuracy can be maximised, a careful examination of the size of gaps between frameworks and casts and where these occur is necessary. Several researchers have studied such spaces in a research context (Sykora, 1997). For example, elastomeric impression material has been allowed to set in gaps and the thickness of the $m$ aterial measured (Stern, et al. 1985). Again, the framework and cast have been encased in resin then sec-

*Corresponding author’s e-mail: rjwillaims@uwic.ac.uk tioned to reveal gaps between the framework and cast, accurately maintained by the resin (Fritell, et al. 1985). Both the latter studies used photographs of sections taken across spaces, which were enlarged by projecting transparencies onto screens to increase accuracy of measurement. Other researchers have shown that a framework may appear to fit 'in vivo' but with the use of, for example, custom made feeler gauges under retentive ends of clasps, have shown that there are gaps not seen with the naked eye (Murray, et al. 1988). The present study suggests that subjective judgments of fit based on the use of the naked eye or experience may be flawed (Calverley, et al. 1987 and Rantanen, et al. 1986).

The above studies, although very suggestive, are unable to show that the fit of a framework on a cast may be in a distorted state (Ali, et al. 1997). Whilst acknowledging that a framework may be distorted when fitted to a cast or indeed for that matter the oral cavity, objectively assessing and measuring spaces between a framework and a cast is an important first step in quality assurance. 
This paper reports on a method of objectively assessing framework fit, which is comparatively fast and efficient and promises to allow accurate measurements of spaces to be made outside the research context on a routine basis.

\section{Methods for Gaps Measurements}

There are three main methods for measuring small gaps. These are X-rays, Ultrasonic and capacitive transducers. The theory of operation of each of these methods is presented below.

\section{$2.1 X$-Ray}

When a material is subjected to $\mathrm{X}$-ray bombardment, some of its electrons will gain energy and leave the atom, creating a void in the vacated shell, thereby releasing a photon of x-ray energy known as $\mathrm{x}$-ray fluorescence.

The energy level or wavelength of fluorescent $x$-rays is proportional to the atomic number and is characteristic for a particular material. The quantity of energy released will be dependent upon the thickness of the material being measured.

Basically, the x-ray fluorescence unit consists of an $x-$ ray tube and a proportional counter. Emitted photons ionize the gas in the counter tube proportional to their energy, permitting spectrum analysis for determination of the material and thickness.

$\mathrm{X}$-ray fluorescence is the most precise measurement method, especially for small-diameter parts, or dual coatings such as gold and nickel over copper.

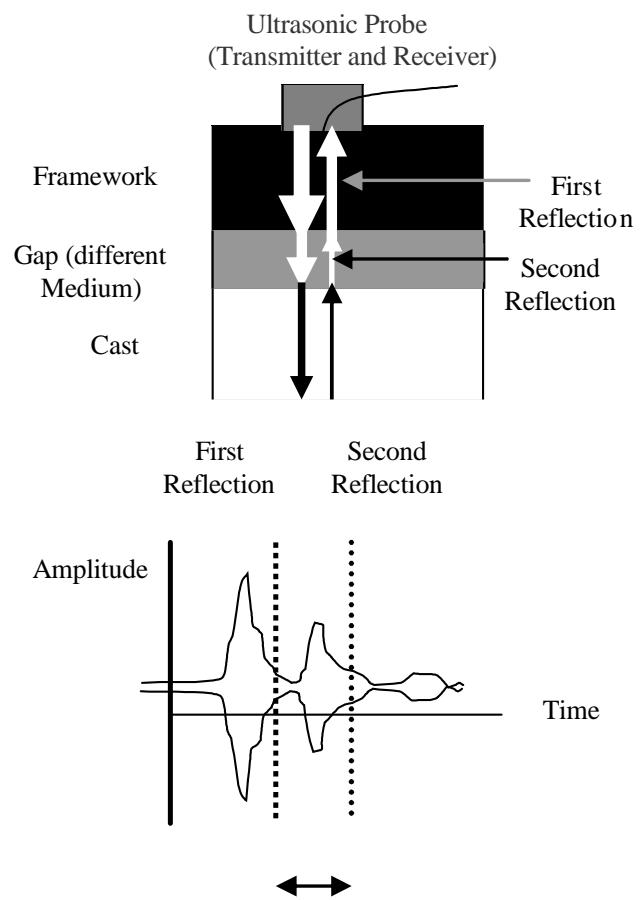

Time difference corresponds to the depth of the gap

Figure 1. Ultrasonic method for measuring the gap between the cast and framework

\subsection{Ultrasonic}

Ultrasonic thickness gauging is a widely used nondestructive test technique for measuring the thickness of a material from one side.

The ultrasonic method uses the pulse reflection principle and is based on the amount of time it takes for ultrasonic waves to pass through and return from inside the object. This is explained below with reference to Fig. 1 .

The measurement process starts by transmitting a pulsed ultrasound energy from the ultrasonic probe through the framework. The ultrasonic pulse will travel through the framework medium until it reaches the interface between the framework and the cast. Because of the difference in the medium, part of the ultrasonic energy will be reflected back to the probe (first reflection) and the majority of the energy will continue travelling through the gap. When the wave reached the end of the gap and hits the cast, another reflection occurs (second reflection). The time between the two reflections depends on the depth of the gap and the sound speed in the medium of the gap.

\section{Gap Depth $=(t 2-t 1) *$ Speed of Sound in the gap}

The resolution of the above ultrasonic method depends on the pulse width (the narrower the pulse, the higher the resolution). Another constraints is the Quality Factor of the ultrasonic probe (minimum number of cycles in the pulse). Typical ultrasonic transducers have a Q-Factor of 10. That means we need to have a minimum of 10 cycles per pulse. To achieve a resolution of $0.1 \mathrm{~mm}$, a very narrow pulse is needed with 10 cycles in it. This means a very high frequency ultrasonic wave is needed (around $100 \mathrm{MHz}$ ). The probes that are capable of working at this frequency are usually expensive and very delicate.

A disadvantage of radiation methods is the use of Xray or gamma radiation that requires special safety measures for protection of the users against the radiation. The equipments used are also relatively expensive.

There were two main limitations for the use of ultrasonic non-destructive testing in this study. These limitations can be summarized as:

1. To achieve a resolution of $0.1 \mathrm{~mm}$ with an ultrasound probe it should have a high frequency. This high frequency depends on the speed of the sound traveling in the material (in this case the Cobalt Chromium RPD framework). The resolution is related to the shortened pulse length that can be produced, which is very difficult to achieve. A demonstration held in the Dental Technology Centre at UWIC was promising but proved very expensive. The technique was excluded due to the high expense of instrumentation needed.

2. An X-ray technique can be used to measure a gap between an RPD framework and a cast. However, about 4 images are needed for each framework and these need to be digitalized before the measurement can be taken. This technique is time consuming. 
Because of the above-mentioned difficulties and disadvantages, an alternative method was sought. Initially it was proposed that the electronic principle of capacitance might be applicable to the problem in hand.

\subsection{Capacitive Transducers}

A capacitor in its simplest form consists of two conducting plates separated by an insulating layer called a dielectric. When a capacitor is connected in a circuit across a voltage source, the voltage forces electrons onto the surface of one plate and pulls electrons off the surface of the other plate resulting in a potential difference between the plates.

The capacitance of a capacitor is proportional to the quantity of charge that can be stored in it for each volt difference in potential between its plates. Mathematically this relationship is written as:

$$
C=Q / V
$$

where $C$ is capacitance in farads, $Q$ is the quantity of stored electrical charge in coulombs and $V$ is the difference in potential in volts.

The difference in potential or voltage of the capacitor can be calculated using the formula:

$$
V=Q / C
$$

The capacitance of a capacitor is affected by three factors:

1. The area of the plates.

2. The distance between the plates.

3. The dielectric constant of the material between the plates.

Larger plates provide greater capacity to store electric charge. Therefore, as the area of the plates increases, capacitance increases.

Capacitance is directly proportional to the electrostatic force field between the plates. This field is stronger when the plates are closer together. Therefore, as the distance between the plates decreases, capacitance increases. As the distance between the plates increases, capacitance decreases.

The ability of the dielectric to support electrostatic forces is directly proportional to the dielectric constant. Therefore, as the dielectric constant increases, capacitance increases.

Taking into account each of the above three factors, the capacitance of a capacitor with two parallel plates can be calculated using the formula:

$$
C=\left(8.855 \times 10^{-12} \mathrm{KA}\right) / d
$$

Where $\mathrm{K}$ is the dielectric constant;

$\mathrm{A}$ is the area of the plate;

$\mathrm{d}$ is the distance between the plates and $8.855 \times 10-12$ is the absolute permittivity of free air space.
Substituting the value of $\mathrm{C}$ described by Eq. 3 in Eq. 2 will result in:

$V=d \times\left(Q / K \times A \times 8.855 \times 10^{-1}\right)$

Equation 4 shows that if Q, $\mathrm{K}$ and $\mathrm{A}$ are kept constant, then the value of the voltage across the two plates are directly and linearly related to the distance between the two plates.

The above principle was used to develop a method of measuring the fit of RPD frameworks to dental casts. The denture is usually made from conductive material and forms one end of a capacitor. The other plate of the capacitor is formed by sticking a small sensor $(0.01 \mathrm{~mm}$ thick) made from conductive foil on the cast. By connecting the ends of these two plates to a high-precision current source and measuring the voltage across the two terminals, since the voltage will be directly related to the gap between the denture and the cast, the distance between the RPD framework and the cast can be determined.

\section{Experimental Set-up and Measurements}

Figure 2 show the experimental set-up used in this project. It is based on a high precision current source developed by Sensatech Research Ltd (Sensatech Research Ltd, Unit 6 Level 3 North, New England House, New England Street Brighton, UK. BN1 4GH, www.sensatech.com). This device is used to maintain constant current (constant Q) in the capacitor, irrespective of its value. The device is powered by 20V DC power supply and the voltage across the capacitor is measured by a digital voltmeter. The voltage should be directly related to the gap between the capacitor's plates.

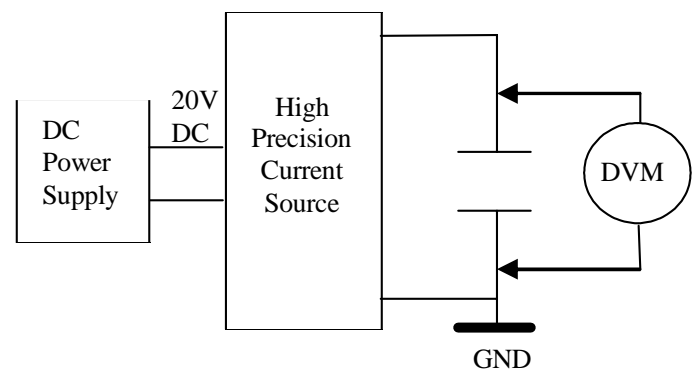

Figure 2. Basic experimental set-up

It was necessary to calibrate the system and find the voltages for different known gaps and for different sensor sizes. To do this, a simple setup was built using a micrometer where one plate of the capacitor was mounted on the fixed end of the micrometer while the other plate (the sensor) was fixed on the moving end as shown in Fig. 3.

The gap between the two plates was varied in $0.1 \mathrm{~mm}$ steps using the micrometer dial and for each gap value the voltage was measured. The results were tabulated in a look-up table and plotted in Fig. 4.

The decision was taken to use one sensor size $(5 \times 5 \mathrm{~mm})$ to carry out the evaluation process. This decision was taken because it has the best straight line and all the RPD components were almost the same size (rest, guide plates, 
terminus third of clasp retentive arms, and the reciprocal clasp arms).

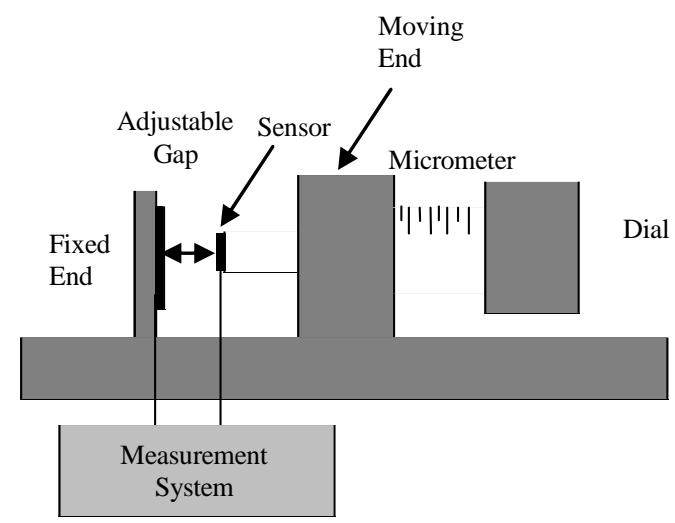

Figure 3. System calibration

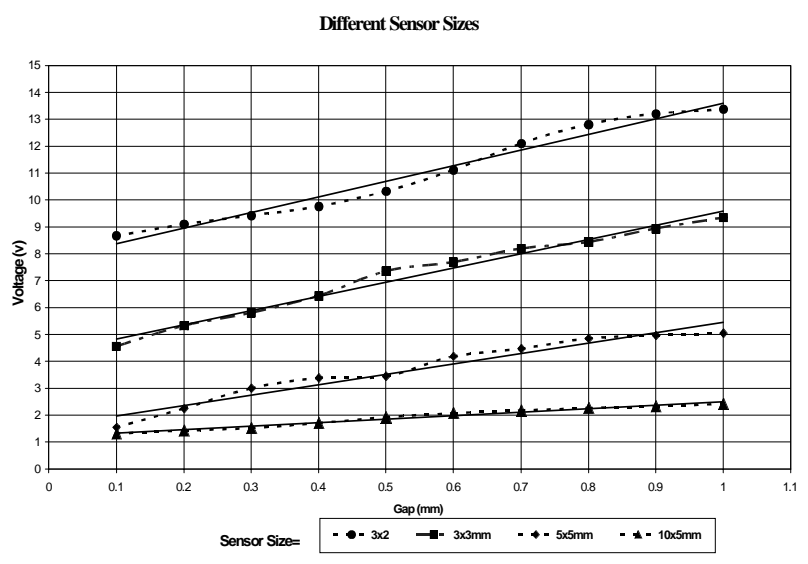

Figure 4. Results of capacitance for known gaps using sensor size $10 \times 5 \mathrm{~mm}, 5 \times 5 \mathrm{~mm}$, $3 \times 3 \mathrm{~mm}$ $3 \times 2 m m$

Sensors were adapted to a cast in critical areas and a framework fitted to the cast. Areas of interest were, for example, under occlusal rests, retentive ends of clasps and guide planes (Fig. 5).

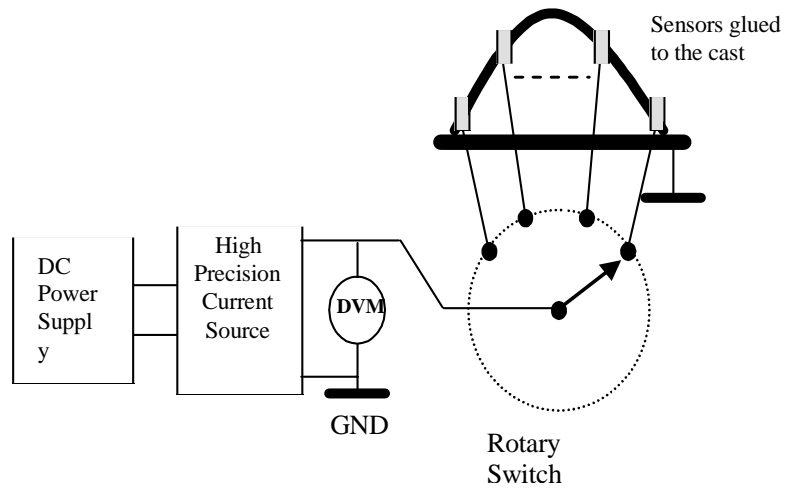

Figure 5. Block diagram of the complete fitness measurement system

The sensors were connected to a circuit shown in Fig. 6. Capacitance readings were taken at each sensor and the size of any gaps determined by using the above graphs.

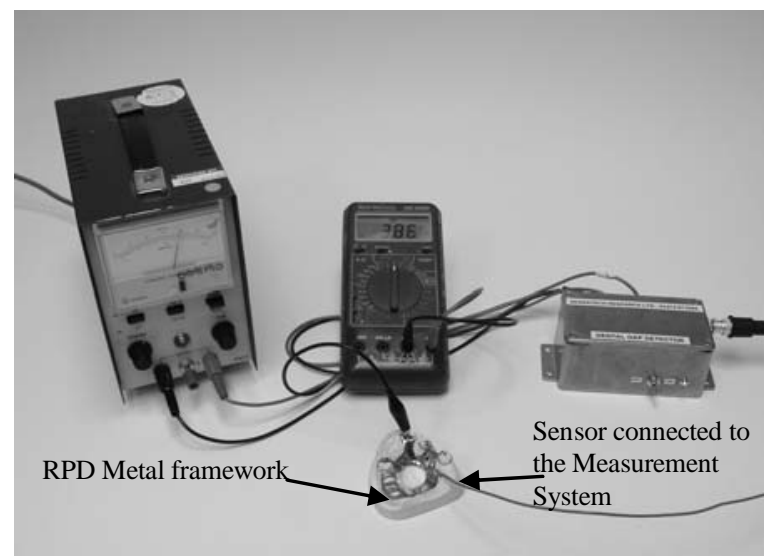

Figure 6. Sensor connected to the dental cast with RPD framework positioned above

The size of gaps measured by capacitance were then verified using radiography (Fig. 6) and by measuring the thickness of acrylic resin usually used for crown and bridge modelling (Palavit ${ }^{\circ}$, Heraeus Kulzer GmbH, Philipp-Reis-StraBe, Germany) material which was allowed to flow into gaps. This technique has been used before to evaluate the accuracy of fitting of RPD framework (Figs. 7a and 7b). The Palavit G resin were placed on the area of concern of the gypsum cast, the framework were then placed to full seating applying sufficient figure pressure allowing the resin to polymerise. The thickness of the resin layer was then measured using digital micrometer calibre. The average of three for each component was then recorded in Table 1. All three methods of measurement yielded results that coincided to a large degree in Table 1. Thus confirmation of the accuracy of the electronic method of measurement described above was provided.

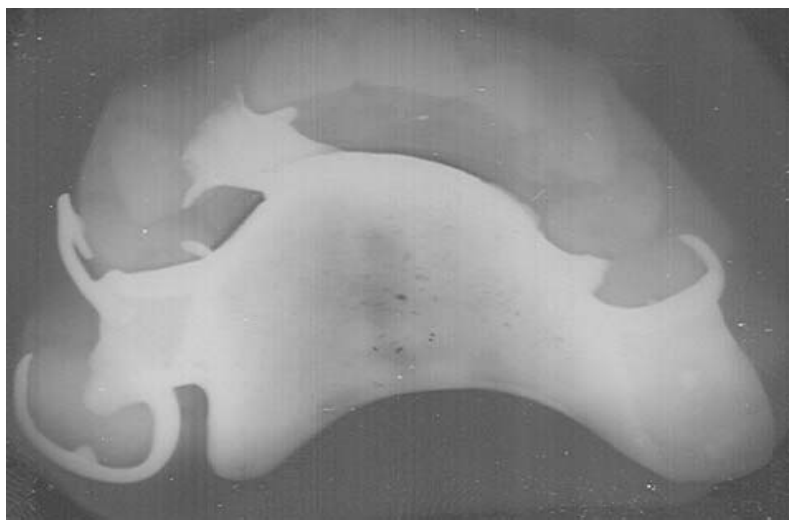

Figure 7a. X-ray image for RPD framework used to confirm the results of the capacitance 


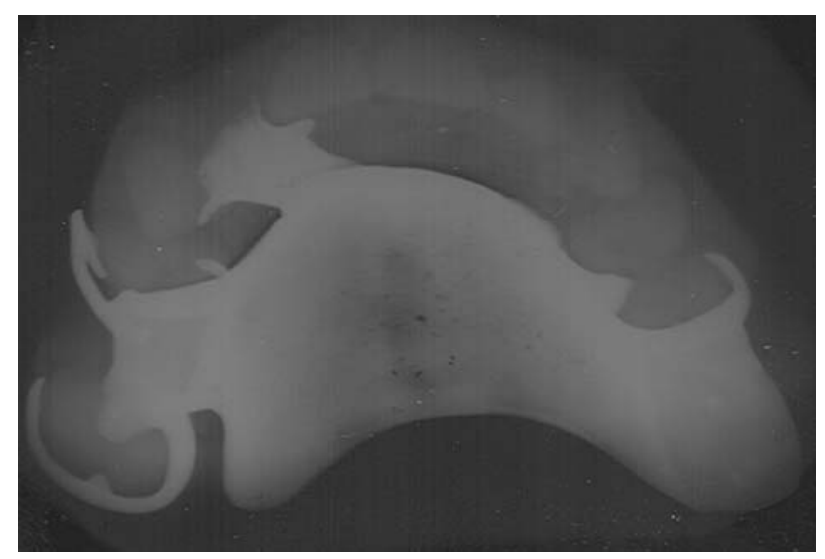

Figure 7b. X-ray image for RPD framework used to confirm the results of the capacitance. It is evident that the gaps appear under the major connector, canine, upper right premolar and upper right molar

\section{Results}

The graph represents the measurements of known gaps. It is shown that the voltage increases as the gap size increases. Area, as well as gap size, affects the capacitance measurement. Thus each line refers to a given area.

The results were verified firstly using X-Rays. A radiograph of gaps between the model and the alloy framework were measured. Secondly a resin (Palavit G) was flowed into gaps, allowed to set and the thickness (corresponding to the gap size) measured

Table 1 shows measurement of gaps using X-Ray, Palavit G, and capacitance measurements. This was achieved by placing the x-ray on a light table, and using the Graticule Magnifier (Lensel Optics Pvt Ltd. 66/2, D2, MIDC, Chinchwad, Pune 411 019, India) with Scale range $0.1 \mathrm{~mm}-22 \mathrm{~mm}$, touching the $\mathrm{x}$-ray image the gap between the metal framework and the cast was measured. Three readings were recorded for each component and the average is shown in Table 1.

Table 1. Comparison of results of measuring the gap between some components of the RPD framework and the supporting cast

$\begin{array}{cccccc}\text { Device } & \text { RetiveAm } & \begin{array}{c}\text { Guide } \\ \text { Plane }\end{array} & \begin{array}{c}\mathrm{M} \mathrm{a} \mathrm{j.} \\ \text { Conn. }\end{array} & \begin{array}{c}\text { Rest }- \\ \text { rest seat }\end{array} & \begin{array}{c}\text { Recip. } \\ \text { Arm }\end{array} \\ \begin{array}{ccccc}\text { Gap on } \\ \text { X-Ray }\end{array} & <0.1 \mathrm{~mm} & 0.2 \mathrm{~mm} & 0.3 \mathrm{~mm} & 0.1 \mathrm{~mm} & <0.1 \mathrm{~mm} \\ \text { Capac. } & 0.0 \mathrm{~mm} & 0.2 \mathrm{~mm} & 0.3 \mathrm{~mm} & 0.1 \mathrm{~mm} & 0.0 \mathrm{~mm} \\ \text { Palavit } \mathbb{R} \mathrm{m} & <0.1 \mathrm{~mm} & 0.15 \mathrm{~mm} & 0.27 \mathrm{~mm} & 0.95 \mathrm{~mm} & <0.1 \mathrm{~mm}\end{array}$

\section{Discussions}

Many clasps engage only a $0.25 \mathrm{~mm}$ undercut. If the terminus of an active clasp presents a gap between itself and the cast of only $0.1 \mathrm{~mm}$, for example, the retentive function of that clasp will be greatly reduced. Similarly, occlusal supports functioning as indirect retainers would not need to move a great distance towards the surface of the underlying tissue to cause lifting of the prosthesis.

The objective measurement of such spaces is an important first step in assessing the function of an RPD. The method described above offers this facility conveniently. Although there is a thickness of sensor and adhesive used in the method described above, this is only $0.16 \%$ of a $0.25 \mathrm{~mm}$ gap, for example. Further, if the sensor is adapted along the whole length of a clasp, it is likely that the clasp will be distorted so as to maintain a similar gap at the active end which would be evident without the sensor. The same is true of occlusal rests if the foil is adapted over the whole rest seat.

The electronic device described above offers a method of simply, accurately and objectively assessing the fit of a framework to a cast in crucial areas, which is readily available and not limited to a research setting. Not only can spaces under important areas of the framework be detected but they can be accurately measured. With the use of the device described, frameworks thought to be satisfactory based on subjective assessment may well be deemed to be unsatisfactory, and vice versa

\section{References}

Ali, M., Narin, R.I., Sherriff, M. and Waters, N.E., 1997, "The Distortion of Cast Cobalt-Chromium Alloy Partial Denture Frameworks Fitted to a Working Cast," J. of Prosthetic Dentistry. Vol. 78(4), pp. 419424.

Brudvik, J.S. and Reimers, D., 1992, "The ToothRemovable Partial Denture Interface," J. of Prosthetic Dentistry, Vol. 68(6), pp. 924-7.

Calverley, M.J. and Moegel, J.R., 1987, "Effect on the Fit of Removable Partial Denture Framework when Master Casts are Treated with Cyanoacrylate," J. of Prosthetic Dentistry, Vol. 58(3), pp. 327-329.

Dunham, D., Brudivik, J., Morris, J., Plummer, K. and Cameron, S., 2006, "A Clinical Investigation of the Fit of Removable Partial Dental Prosthesis Clasp Assemblies," J. of Prosthetic Dentistry, Vol. 95(4), pp. 323-6.

Fritell, D.N., Muncheryan, A.M. and Green, A.J., 1985, "Laboratory Accuracy in Casting Removable Partial Denture Frameworks," J. of Prosthetic Dentistry, Vol. 54(6), pp. 856-862.

Massad, D., William, A. and Lily, T., 2006. "A Method to Minimize RPD Framework Deformation During Fabrication Procedure," Dentistry Today, Vol. 25(2), pp. 122, 124, 126.

Murray, M.D. and Oyson, J.E., 1988, "A Study of Clinical Fit of Cast Cobalt-Chromium Clasps," J. of Dentistry, Vol. 16(3), pp. 135-39.

Rantanen, T. and Eerikainen, E., 1986, "Accuracy of Palatal Plate of Removable Partial Dentures, and Influence of Laboratory Handling of the Investment 
on the Accuracy," J. of Dental Materials, Vol. 2, pp. 28-31.

Stern, M.A., Brudvik, J.S. and Frank, R.P., 1985, "Clinical Evaluation of Removable Partial Denture Rest Seat Adaptation," J. of Prosthetic Dentistry, Vol. 53(5), pp. 658-62.

Sykora, O. and Sutow, E.J., 1997, "Improved Fit of Maxillary Complete Dentures Processed on High Expansion Stone Casts," J. of Prosthetic Dentistry, Vol. 77(2), pp. 205-8, 